Mots. Les langages du politique

$83 \mid 2007$

Dire la démocratie aujourd'hui

\title{
Quand les mots de l'abstention parlent des maux de la démocratie
}

Denis Barbet

\section{(2) OpenEdition \\ Journals}

Édition électronique

URL : https://journals.openedition.org/mots/890

DOI : $10.4000 /$ mots.890

ISSN : 1960-6001

Éditeur

ENS Éditions

Édition imprimée

Date de publication : 1 mars 2007

Pagination : 53-67

ISBN : 978-2-84788-111-0

ISSN : 0243-6450

\section{Référence électronique}

Denis Barbet, "Quand les mots de l'abstention parlent des maux de la démocratie », Mots. Les

langages du politique [En ligne], 83 | 2007, mis en ligne le 01 mars 2009, consulté le 22 avril 2022. URL : http://journals.openedition.org/mots/890 ; DOI : https://doi.org/10.4000/mots.890 


\section{Quand les mots de l'abstention parlent des maux de la démocratie}

La fréquence de l'abstention a souvent été considérée comme l'un des principaux indicateurs d'une « crise » de la démocratie représentative. Ce diagnostic, parfois dénié ou euphémisé, véritable serpent de mer du commentaire politique $^{1}$, s'impose aujourd'hui comme une évidence et envahit le discours électoral. Nous avons choisi de privilégier une approche lexicale de l'abstention. Étudier l'expression permet d'accéder aux représentations sociales du vote et de son «négatif». Même si, du fait de l'origine des discours étudiés (ici, ceux des professionnels de la politique, de l'analyse et du commentaire des élections), l'entrée lexicologique nous livre d'abord des indications sur les conceptions d'élites spécialisées.

Dominique Memmi (1985, p. 319 s.) avait décelé en France notamment, à partir des années soixante-dix, une distanciation progressive de la science politique à l'égard de la composante normative de la participation. Est-ce à dire que toute tentation moraliste ou normativiste (Offerlé, 1987, p.7-8; Braud, 1980, p. 17) aurait disparu des propos, notamment "scientifiques », sur l'abstention? Que les commentateurs s'en tiendraient aux simples constat, mesure et analyse du phénomène ? Certes, sur le long terme, une tendance générale à «l'allègement de la pression sociale contre l'abstention » (Duchesne, 1994, p. 186), qui coïncide avec l'entrée dans un cycle de déclin participatif à partir des années quatre-vingt, est perceptible dans différents discours : dans les dictionnaires, révélateurs à la fois de l'état de la langue et des perceptions sociales en vigueur (ils ont été durablement de véritables manuels de la morale républicaine), mais aussi dans la presse et la littérature électorales. L'abstention est globalement moins stigmatisée qu'autrefois. Pourtant, dans chacun de ces domaines, cette évolution doit être nuancée :

1. Le publiciste Charles Benoist intitulait à la fin du $19^{\mathrm{e}}$ siècle un ouvrage Crise de l'État moderne (De l'organisation du suffrage universel) [1895, Paris, Firmin-Didot], et l'AFSP consacrait en 1960 une table ronde à la « dépolitisation » des Français. Nous remercions Paul Bacot, qui avait déjà repéré nombre des expressions mentionnées ici dans son Dictionnaire du vote, ainsi que MarieLuce Honeste et Philippe Corcuff, pour leurs constructives remarques.

Institut d'études politique de Lyon, CNRS (Triangle), denis.barbet@univ-lyon2.fr 
- La comparaison dans le temps des dictionnaires de langue française atteste ce déclin de la normativité. Le contraste est flagrant entre la réprobation exprimée par les Larousse de 1866 à $1960^{2}$, et la définition plus distanciée de l'abstentionnisme, tardivement adoptée en 1982 (vol.1, p. 32) : «nonparticipation, volontaire ou non, à une élection ou à un référendum $»^{3}$. Bien sûr, les dictionnaires aident aussi à comprendre les discours contemporains sur l'abstention, par leurs informations définitoires et étymologiques. Dans les langues romanes, la souche abstinere (tenir éloigné; abs : hors et tenere: tenir ${ }^{4}$ ), a finalement donné abstinence et abstention, formes à l'origine concurrentes $^{5}$. La première conservera le sens de privation, la seconde prenant peu à peu une signification plus politique, sans renoncement à l'idée de privation volontaire (du droit de vote). L'origine religieuse (et stoïcienne) des mots de l'abstention ${ }^{6}$ contribue à éclairer le traitement souvent moral de l'objet. Enfin, les lexies de l'abstention ont plutôt été rangées par la langue du côté de l'inaction, opposées à l'activité du vote. Même pour le Robert de 1951, pourtant peu moraliste, les traits passifs restent dominants : s'abstenir, c'est « s'empêcher [...] ou négliger de faire quelque chose / ne pas agir / rester neutre. Antonymes : agir... » (p.19). Et même quand l'abstention a pu être définie comme une "action» (comme tous les mots en -tion, elle dénote un acte ou son résultat), elle tend le plus souvent à être non seulement «inactivée», mais aussi délégitimée.

- L'évolution est similaire dans le discours de presse. Pour ne retenir qu'un exemple dans la presse quotidienne régionale, le Progrès de Lyon, de la Libération aux années soixante-dix, publie à la une de pressants appels à la «mobilisation» (Votez dimanche!), en multipliant les leçons de civisme (ex: «Dans une démocratie, l'abstention est toujours une faute grave qui conduit aux pires catastrophes. Il faut voter », 25-26 avril 1953). De telles injonctions ne s'estomperont qu'au cours des années quatre-vingt. Même si cette mutation a connu

2. Le Larousse 1866 (rééd. 1990, p. 37) associe l'abstention (« renonciation des électeurs à l'exercice du droit de suffrage ») à la «négligence » et la condamne « d'une façon absolue comme un égoïste oubli des devoirs envers la société ». Les versions de 1922 et de 1928 ajoutent le blâme (p. 9 et p. 23); celle de 1960 «le désir d’éluder une responsabilité » (p. 29).

3. Le Robert avait adopté dès 1970 une définition neutre : «Attitude politique de quelqu'un qui ne vote pas » (Le supplément, p. 3).

4. Le Trésor de la langue française (désormais: TLF) signale comme valeurs originelles de s'abstenir : «(se) tenir à l'écart» et « se priver volontairement de certains plaisirs» (1986, p. 256-257). Les mots de l'abstention portent d'emblée en français une valeur moins « objective » que dans les langues germaniques: en anglais, on dit non-voter et en allemand Nichtwähler (la personne ne vote pas, c'est tout). En français, on ne dit pas les non-votants, alors qu'on parle des non-inscrits, et abstentionniste suggère que la personne n'est pas là où elle pourrait (... et devrait) être.

5. Le mot abstinent a été utilisé notamment par Proudhon dans un sens politique, plus positif qu'abstentionniste (Larousse, 1866, p. 37). Le substantif abstenant(ante) a aussi été employé (ibid. ; TLF, 1986, p. 255).

6. S'abstenir et abstention n'acquièrent un sens politique secondaire qu'au milieu du $19^{\mathrm{e}}$ siècle (en 1866 et 1863), alors que les dérivés abstentionniste (1863) et abstentionnisme (1870) sont d'emblée électoralisés. 
dans la presse des temporalités diverses, les journalistes pourront désormais manifester plus facilement une compréhension des abstentionnistes, leur donner la parole et attribuer une partie des «torts » aux politiciens. Leur style devient moins moralisateur, même s'il subsiste une part de culpabilisation. Et certaines conjonctures, comme celle de l'après 21 avril 2002, voient refleurir les semonces médiatiques, motivées par le «danger lepéniste », y compris dans des publications d'ordinaire peu électoralistes (comme Charlie-Hebdo).

- La littérature politologique, enfin, a connu une tendance analogue. Après l'avancée « objective » permise par Alain Lancelot en 1968 (L'abstentionnisme électoral en France), après les lectures critiques de la participation (Gaxie, 1978 ; Braud, 1980), un traitement moins normatif de l'abstention s'est enraciné dans la discipline. Il culmine avec les approches socio-historiques du vote. Pourtant, nous le verrons, la tentation prescriptive persiste dans certaines analyses, par le biais de présupposés normatifs, de jugements langagiers implicites, voire de fortes sentences.

C'est en considérant l'abstention et la démocratie sans préjugé, en leur réservant un regard "distancié » (Braud, 1980, p. 17; Garrigou, 2002, p. 8-9), que nous étudierons les discours professionnels contemporains des politologues, des journalistes et des politiques. Comme nous recherchons des énoncés dans plusieurs types de discours, le corpus est délibérément hétérogène et large ${ }^{7}$. Nous laisserons de côté les simples traductions statistiques ou évaluatives du phénomène (taux plus ou moins forts), pour nous intéresser à toutes les autres manières de dire l'abstention ou les maux de la démocratie.

Afin d'ordonner la masse des usages lexicaux (les limites de cet article contraignant par ailleurs à réduire l'exemplification), différents champs métaphoriques seront distingués. Les mots et syntagmes liés à l'espace seront évoqués avant d'autres tropes, renvoyant à différents domaines-sources. Néanmoins, tous ces registres restent liés: par exemple, la lexie démobilisé est indissolublement martiale, spatiale et affective. D’ailleurs, la réussite sociolexicale des figures ici analysées doit sans doute beaucoup à leur polysémie.

Certes, les mots et expressions peuvent être utilisés dans des buts différents. Mais même si un locuteur cherche simplement à styliser ou orner son discours pour éviter une répétition, ou bien cède à des automatismes langagiers,

7. Il résulte de l'exploitation secondaire d'un corpus général utilisé pour une étude de la figure halieutique de l'abstentionniste. Ont été explorés quinze dictionnaires des $19^{\mathrm{e}}$ et $20^{\mathrm{e}}$ siècles (neuf cités ici) ; pour la production savante : la plupart des ouvrages et articles majeurs en langue française depuis 1965, sur l'abstention et les comportements électoraux (une vingtaine cités ici), ainsi que quelques interventions politologiques médiatisées ; pour le discours journalistique, surtout la presse écrite et, systématiquement, les commentaires électoraux de la presse nationale des lendemains de scrutins en particulier (L'Aurore, Le Figaro, L'Humanité, Libération, Le Monde, depuis 1968), des quotidiens régionaux (notamment lyonnais: Le Progrès depuis 1936), des hebdomadaires (Le Point, Marianne, Le Nouvel Observateur, depuis 1995 et l'intégrale du Canard enchaîné). Le discours politicien cité est celui rapporté par les médias. 
ces façons de parler nous paraissent révélatrices. Le lexique est souvent normatif et l'abstention n'est pas située du « bon » côté.

\section{L’abstention en négatif : des clivages très spatiaux}

Quelques couples d’antonymes traduisent une position peu valorisée.

\section{Bas / haut}

En règle générale, l'abstention est plutôt en bas ; sa progression est associée à un mouvement montant et l'on évoque alors une chute de la participation. Pour ne retenir qu'un exemple, politologique : «Le premier [signe de la crise] était la montée de l'abstentionnisme lors des élections législatives [...]. L'élection présidentielle, elle-même, n'échappait pas à la règle puisque la participation baissait [...] en 1995 » (Perrineau, Ysmal, 2003, p. 15).

Déjà, ces orientations spatiales sont instructives : la montée peut servir à désigner dans différents domaines des progressions anormales, menaçantes ou catastrophiques, sur le schéma conceptuel de la crue (Honeste, 2000); la chute renvoie alors à d'autres expériences négatives, physiques ou morales. Sur cet axe vertical, où une axiologie fortement prononcée dévalue le bas (RémiGiraud, 2005, p. 99), l'abstention peut entrainer également vers le fond : « Cette chute de la participation [...] aura révélé la profondeur de la crise de la représentation politique » (Jean-Paul Monferran, L'Humanité, 17 juin 2002).

Quand l'abstention diminue, comme entre les deux tours de la présidentielle de 2002, politologues et journalistes parlent parfois de sursaut ${ }^{8}$ (démocratique, civique, républicain) : «Ce sursaut civique a concerné tous les âges » (Clanché, 2003, p. 2). Ou bien d'élan (mouvement plutôt vers l'avant). Mais comme les législatives suivantes infirment la tendance, Philippe Broussard et ses confrères titrent leur article «L'élan perdu du 5 mai » (Le Monde, 11 juin 2002).

\section{Périphérie / centre}

Chez les politologues en particulier, l'abstention est à la participation ce que la périphérie est au centre et le lointain au proche: «[l'élection] ne remet pas totalement en cause la répartition géographique de l'abstention entre départements civiques et départements “distants” à l'égard de la participation politique » (Perrineau, Ysmal, 1995, p. 14) ; « un nombre croissant d'électeurs manifestaient leur éloignement par rapport au système politique [...]» (Perrineau, Ysmal, 2003, p. 16).

8. À la fois redressement et effort : Le Nouveau Petit Robert 1 (désormais : PR), 1993, p. 2182. 


\section{Dehors /dedans}

L'abstention tend à être située dehors: «Plus du tiers des Français [...] sont restés en dehors de la décision électorale» (Muxel, 2003, p.153). Le choix même du mot hors-jeu, abondamment utilisé par la spécialiste Anne Muxel à partir de 1997 pour caractériser une variante de l'abstention (par désintérêt ou éloignement partisan), n'est pas sans portée normative. Faut-il rappeler que dans le domaine sportif, le hors-jeu désigne la « faute d'un joueur, dont la position sur le terrain est interdite par les règles » $(P R$, p. 1 103)?

Plusieurs expressions plus ou moins figées viennent confirmer cette première impression négative.

\section{Le retrait et le refuge dans l'abstention}

Le mot retrait, en partie explicatif, d'usage assez récent et surtout politologique, désigne «le fait de se retirer» mais aussi de « revenir en arrière » $(P R$, p. 1 969).

Il place encore l'abstentionniste derrière ou à côté : «Le sentiment de l'inutilité du vote [vient] encore renforcer le retrait à l'égard du politique » (Subileau, 1992, p. 29); « [...] ceux qui sont restés en retrait de l'élection » (Muxel, 2005, p. 74).

La métaphore du refuge, au sens de lieu « où se rassemblent des personnes qui ne peuvent ou ne veulent pas aller ailleurs » $(P R$, p. 1904), inspire aussi les électoralistes : «Une fraction des poujadistes s'est réfugiée dans l'abstention » (Goguel, 1983, p. 35, législatives de 1958); « refuge dans l'abstention » ou « se réfugier dans l'abstention » (Subileau, 1992, p. 26, 41 et 1996, p. 147, 151).

Pourquoi préférer cette notion, sinon pour évoquer le retrait, voire une forme de démission, au simple «choix de s'abstenir», locution certes moins imagée mais aussi moins dépréciative? En tout cas, l'abondant recours des politologues aux métaphores spatiales contribue à situer l'abstention aux antipodes d'une scène électorale centrale, légitime, et d'une participation supposée normale.

\section{Le chemin des urnes}

D'autres locutions indiquent le «bon » chemin, celui qui mène au vote : trouver / retrouver / prendre / reprendre le chemin des urnes ou des bureaux de vote, ramenervers les urnes. Ces expressions suggèrent la règle, tandis que les abstentionnistes sont supposés s'égarer en adoptant d'autres destinations dominicales. Pour ne retenir que deux énoncés: un journaliste précise après les européennes de 1999 que « les électeurs se détournent des urnes» (Alain Dauvergne, Le Point, 4 juin). En 2002, un candidat déclare: «l'espère que pour le 
second tour nous pourrons ramener vers les urnes ces électeurs » (Daniel Paris [PS-MRG], L'Yonne Républicaine, 10 juin).

\section{Voter avec ses pieds}

Cette curieuse formule désigne l'évitement des urnes. Le 20 mars 2004, veille des élections régionales, André Santini, tête de liste francilien de l'UDF, déclare ainsi sur France Info: "Si nous n'étions pas là, les électeurs voteraient avec leurs pieds, ils s'abstiendraient. » L'expression, dont Yves Schemeil (2001, p. 61) restitue l'origine antique, emporte peu de péjoration. Elle est même plutôt active et renvoie à une forme alternative d'engagement. Elle peut quasiment être entendue au sens anatomique : on vote avec ses pieds plutôt... qu'avec ses mains. Notons toutefois que le langage courant oppose le geste adroit prototypiquement effectué par la main à l'action maladroite (agir comme un pied).

\section{Des verbes de mouvement diversement connotés}

Voter (sauf par procuration) suppose un déplacement de l'électeur. L'abstentionniste, lui, ne va pas voter, ne se déplace pas, ne se présente pas auxurnes. La plupart de ces phrases, bien que négatives, restent plutôt constatives, comme : « La moitié des dix-neuf millions d'électeurs appelés à élire les conseillers généraux [...] ne s'est pas déplacée » (Lyon-Matin, 29 septembre 1988). Pourtant, opposer l'électeur mobile à l'abstentionniste statique n'est pas anodin dans une société valorisant activité et mouvement. L'inertie souvent caricaturée de l'abstentionniste, soupçonné de rester chez lui ou immobile au bord de l'eau en lorgnant le bouchon de sa ligne, traduit une certaine dépréciation. Et la péjoration devient manifeste lorsque la carence de geste civique est liée à une négligence citoyenne (souvent précisée par l'environnement verbal). C'est le cas dans l'énoncé politologique suivant: «Les abstentionnistes “dans le jeu” ne se différencient guère de l'ensemble de ceux de leurs concitoyens qui n'ont pas pris la peine d'aller voter » (Capdevielle, 2005, p. 47). La réprobation journalistique est aussi marquée: "11,6 millions d'électeurs n’avaient pas daigné se déplacer » (Gilles Drevet, Le Progrès, 6 mai 2002) - la démocratie méritant des égards.

Une autre expression, se rendre aux urnes, très fréquente dans le commentaire savant et médiatique, apparait assez neutre, bien qu'elle se traduise par un mouvement plutôt volontaire ${ }^{9}$. Le voisinage lexical peut alors renseigner sur la cible ou l'analyse de l'énonciateur. Dans l'extrait politologique suivant, ce ne sont pas les abstentionnistes qui sont incriminés, même si la bonne disposition

9. Se rendre signifie, secondairement: "aller, se transporter », mais aussi, premièrement: "se soumettre, céder» (PR, p. 1928). 
du citoyen déterminé est postulée : « Les électeurs du camp battu [...] pourraient bien rechigner à se rendre aux urnes » (Pascal Perrineau, cité par Le Monde, 1718 mars 2002). Le paratexte vient parfois renforcer la représentation obligatoire et solennelle du vote, comme pour cet énoncé de L'Aurore du 24 juin 1968 : «C'est dans la plus parfaite sérénité que les électeurs se sont rendus aux urnes. Ici, comme partout ailleurs, la participation a été plus importante que l'an dernier. » Le titre général, sur six colonnes ("Participation massive au scrutin »), souligne le contraste recherché entre la force et la légitimité du peuple paisible et souverain, et l'effervescence de la rue durant les semaines précédentes.

\section{Les figures du manque, de l'absence et de la vacuité}

L'abstentionnisme est parfois décrit comme un manque. Manque d'électeurs bien sûr, mais aussi défaut d'assiduité, de la part des mauvais élèves de la démocratie: «L'est lyonnais vient tout particulièrement de se faire remarquer par son manque d'assiduité » (Manuel da Fonseca, Lyon-Matin, 7 juin 1988). L'assiduité désignant une « présence régulière en un lieu où l'on s'acquitte de ses obligations » (PR, p. 137), aspects moral et spatial sont encore associés. Et la dénonciation devient plus claire quand on passe du manque au manquement $(P R$, p. 1346) : "C'en était fini, croyait-on, des manquements citoyens » (Philippe Broussard, Le Monde, 11 juin 2002).

D’autres énoncés disent l'absence, comme le suivant, qui répartit les torts : "L'absentéisme des électeurs répondait-il à la vacuité, voire l'absence totale, du débat de fond ? " (Jean-Philippe Mestre, Le Progrès, 17 juin 2002). Cela étant, on peut s'étonner, tant ils paraissent adaptés à l'état du «non-votant $»^{10}$, de la relative rareté dans le corpus des mots du champ de l'absence. Sont-ils lexicalement trop proches d'abstention? Ou bien le constat de la carence de l'électeur est-il trop banal au regard des conjectures sur ses occupations, halieutiques ou domestiques?

\section{Des tropes non spatiaux pour dire l'abstention}

Une pluralité d'autres registres tropiques sont sollicités :

\section{Des images sportives}

Un vocabulaire sportif vient couramment ponctuer les commentaires sur la participation: "Le scrutin du 9 juin a été marqué par un record historique [...] » (Muxel, 2003, p. 1) ; «L'abstention [...] n'a pas battu, avec 30,94\%, le record de la Ve République » (Pierre Viansson-Ponté, Le Monde, 17 juin 1969).

10. Absent : « qui n'est pas (dans le lieu où il pourrait, devrait être), qui [en] est éloigné » (PR, p. 9). 
Ces métaphores sont encouragées dans le discours médiatique et politique par l'actualité sportive, comme en 1988 (J. O. de Séoul et cantonales) : "S’il existait des Olympiades de l'abstention, nous serions médaille d'or » (RogerGérard Schwartzenberg [MRG], Le Monde, 27 septembre 1988). Pourtant, record désignant « un exploit sportif» $(P R$, p. 1891$)$, les scripteurs éprouvent quelque gêne à qualifier favorablement l'abstention. Des adjectifs affectifs et axiologiques, souvent antéposés, parfois mis entre parenthèses, sont alors employés pour péjorer la «performance » en question : «Un (triste) record pour le département » (Lyon-Figaro, 6 juin 1988) ; «le record peu glorieux de taux d'abstention » (Le Progrès, 26 septembre 1988) ; " [l'abstention] a hier, une fois encore, été la triste vedette de la soirée » (Le Progrès, 3 octobre 1988).

\section{Des représentations pathologiques}

Le traitement de l'abstention comme un phénomène morbide est évidemment à rapporter au diagnostic pointant la «crise » de la démocratie: "Après les élections des parlementaires européens, chacun s'accorde à reconnaître que la démocratie représentative est en crise [...]. On retrouve un peu partout les mêmes symptômes " (Capdevielle, 2005, p. 10).

Parmi les troubles récurrents du citoyen, on trouve par exemple l'apathie, l'anémie, l'asthénie (démocratique), l'atonie, la dépression (électorale) ou la fracture (civique). Pour ne retenir que l'apathie, fréquente et ancienne ${ }^{11}$, elle ressortit aux champs affectif (avec une connotation morale) et médical: "Les recettes contre l'apathie électorale» (Lyon-Libération, 26 septembre 1988). Et lorsque la nosographie ne suffit plus, des maladies sont inventées, comme l'urnophobie $^{12}$ (ironiquement) ou l'abstention(n)ite $e^{13}$, déjà signalée par le TLF en 1959, dont un exemple métaphoriquement filé apparait dans la presse lyonnaise :

Le virus de "l'abstentionite» a fait des ravages sans distinction d'appartenance politique dans les rangs des électeurs [...]. Si les électeurs souffrent d'« overdose» de scrutins, les candidats sont, eux, confrontés à l'état de manque... en pourcentages! Un bien mauvais bulletin de santé pour le corps politique départemental. (JeanJacques Billon, Lyon-Matin, 27 septembre 1988)

Sans qu'il s'agisse vraiment de pathologie, une place doit être accordée ici au champ sémantique du sommeil, également fréquent et ancien. Quand l'abstention est liée à la fatigue, à la lassitude ou au sommeil du «corps électoral»,

11. Voir Le Progrès du 14 octobre 1946. Apathie est abondamment utilisée par Meynaud et Lancelot (1965).

12. Le Canard enchaîné, 28 septembre 1988.

13. «Tendance, considérée comme une maladie du corps social, à pratiquer l'abstention » (TLF, p. 258). 
le registre est physiologique. Mais ces états de l'organisme peuvent être dénoncés comme de mauvais prétextes, symptomatiques d'une paresse citoyenne, évidemment plus propice à la réprobation. Les journalistes français, comme les caricaturistes et leurs confrères étrangers ${ }^{14}$, recourent largement à cette image de l'assoupissement: «Il faut réveiller en sursaut les électeurs et, pour les tenir éveillés, il est préférable de parler "patrie en danger" [...] 》 (Serge July, Libération, 28 avril 1997) ; "Pourvu que les abstentionnistes se réveillent » (Le Monde, 7 mai 2002).

L'électeur n'apparait pas dans une forme olympique, pour cet acteur qui ne recule devant aucun amalgame politisant: «On nous dit que les Français sont fatigués d'aller voter, mais ils le sont aussi d'aller à la messe, au cinéma et sur les terrains de football. Ils refusent l'effort et les résultats de Séoul révèlent un peuple affaibli » (Christian Bonnet, UDF, cité par Le Monde, 27 septembre 1988).

\section{Des figures martiales}

Le vocabulaire militaire marque aussi une partie de la rhétorique sur l'abstention. Certains mots relèvent de ce lexique, mais renvoient aussi, par exemple, à l'espace (avec parfois une dimension morale). La figure saillante de la mobilisation électorale est ainsi diversement déclinée : mobiliser, se mobiliser, se démobiliser, être démobilisé... Que les candidats soient friands de ces formules, pour appeler l'électorat à les soutenir, n'est guère surprenant: « Tout est encore possible, à condition de mobiliser les abstentionnistes » (Élisabeth Guigou [PS], Le Progrès, 10 juin 2002). Mais les journalistes en usent aussi abondamment: "La Manche a connu une forte démobilisation de son électorat» (Le Monde, 27 septembre 1988). Comme les politologues: «[...] la campagne a démobilisé l'électorat» (Perrineau, Ysmal, 2003, p. 19).

La métaphore des troupes est également convoquée, en particulier entre les deux tours, dans les termes spéculatifs de réserves utiles à la victoire : «La gauche a des réserves [...]. Toute la gauche, tous ceux et toutes celles qui se refusent à voir la France revenir en arrière doivent donc se mobiliser » (déclaration du bureau politique du PCF reproduite par Le Monde, 17 mars 1982).

Enfin, la vieille thématique ${ }^{15}$ de la désertion des abstentionnistes (parfois liée à une dette, dont l'électeur doit s'acquitter, envers des ancêtres qui se sont battus pour le droit de vote ${ }^{16}$ ) est à la fois martiale, morale (trahison d'un devoir) et spatiale (le vide). Une analyse distributionnelle montre ainsi des

\footnotetext{
14. Les Allemands emploient couramment le mot Wahlmüdigkeit (fatigue de voter) ; les Scandinaves parlent du Sofavelger (celui qui vote pour son canapé) pour moquer l'abstentionniste.

15. Voir Gueniffey, 1993, p. 191.

16. Vision démentie, en France en tout cas, par l'observation historique (Garrigou, 2002, p. 8-9, note 3).
} 
électeurs désertant les urnes, les bureaux de vote, les isoloirs, le scrutin... Cette figure très stigmatisante est le plus souvent journalistique, mais aussi savante : "Voter est un devoir. S'abstenir c'est déserter » (Le Progrès, 8 novembre 1946); "Les déserteurs du scrutin représentent $36 \%$ des inscrits» (François Darras, Marianne, 17 juin 2002) ; "La démocratie [...] se voit frappée par un mouvement de désertion civique [...] » (Marcel Gauchet, cité par Le Monde, 18 juin 2002).

\section{Des tropes affectifs}

On ne s'étonnera pas de rencontrer un vocabulaire émotionnel ou psychoaffectif (Braud, 1996) à propos d'un acte aussi solennisé que le vote, et pour désigner la relation à des représentants dont la légitimité repose précisément sur l'attachement des citoyens. L'abstention est mise en scène à travers une description des états d'âme de l'électeur, qui constituent, pour les journalistes en particulier, une amorce interprétative, au moins psychologique. On trouve ainsi, par ordre de fréquence décroissante : la désaffection, le désintérêt, l'indifférence, le manque de passion, d'enthousiasme, de zèle, l'insouciance ou le désenchantement. Par exemple: "La désaffection guette ce scrutin de 1997 » (Serge July, Libération, 28 avril 1997) ; « abstentionnistes [...] qui se désintéressent du sort de la France» (Florence Assouline, Marianne, 17 juin 2002); «On a voté sans trop d'enthousiasme » (J. de Coulonces, L'Aurore politique, 24 avril 1972) ; "désenchantement démocratique» (le politologue Philippe Méchet, TF1, 16 juin 2002, 19 h 40).

Avec le mot renonciation, on retrouve le sens originel (voir supra) de s'abstenir. Mais cette attitude n'est pas toujours comprise d'analystes ayant du mal à se résoudre à l'idée que des Français puissent se montrer plus insensibles qu'eux à la politique : « Plus grave encore, le désintérêt pour la réforme du quinquennat recèle une forme de renonciation des électeurs à l'un de leurs pouvoirs » (lérôme Jaffré, «La mauvaise affaire du référendum », Le Monde, 21 septembre 2000). L'une des images affectives les plus présentes reste celle de la bouderie ${ }^{17}$ : «Les boudeurs d'urnes du premier tour se situent pour beaucoup dans l'électorat de gauche » (Alain Raynal, L'Humanité, 15 mars 2001).

D'abord médiatique, cette tournure figure aussi dans la production savante. Elle est omniprésente depuis les années 1980, sous différentes formes, notamment métonymiques : l'électorat boude les urnes; les Français ou les citoyens boudent la politique; les électeurs boudent les élections / les bureaux de vote / les isoloirs, etc. Ainsi, l'essayiste et journaliste Alain Duhamel réprimande les abstentionnistes dans un article de 1990, éloquemment titré " Les enfants gâtés de la démocratie. Quand les Français cesseront-ils de bouder la politique?»:

17. Bouder: « montrer du mécontentement par une attitude [...] que l'on entretient à dessein » $(P R$, p. 246). 
«Alors que partout en Europe de l'Est, les peuples se battent pour arracher leurs libertés [...], ici, dans le pays qui s'enorgueillit d'être le berceau des droits de l'homme, les citoyens boudent la politique. Il y a dans ce contraste brutal [...] quelque chose de presque inconvenant » (Le Monde, 21-22 janvier 1990).

\section{De la «grève des électeurs»}

Une place à part doit être réservée à la métaphore de la grève des électeurs ou des urnes, plus difficilement classable. Son interprétation demande d'envisager le contexte. Elle n'est pas forcément péjorative. Elle ne l'était assurément pas quand elle permettait à Octave Mirbeau, en 1888, de dénoncer le suffrage universel et ses utilisateurs crédules (Le Figaro du 28 novembre: Mirbeau, rééd. 1995).

De même, en 2000 , trotskistes et communistes ${ }^{18}$ utilisent positivement l'expression, la grève faisant partie de leur culture militante. En revanche, la connotation semble moins appréciative lorsque Gérard Grunberg, par exemple, intitule un de ses articles, en 1989: «La grève des urnes ». Légitimant la participation, l'auteur conclut en effet, comme pour nous rassurer à propos de l'abstention : «Un tel climat n’est pas nécessairement durable » (p. 132).

\section{Des cibles diverses, des degrés et formes variables de jugement moral}

Bien sûr, ces façons de parler de l'abstention engagent des jugements de valeur d'intensité variable. Les différents énoncés montrent par ailleurs que les cibles des locuteurs ne sont pas toujours les seuls abstentionnistes. Les journalistes par exemple peuvent leur faire la morale, mais aussi dans certains cas, et même simultanément, interpeller la classe politique, en partageant les réserves de leurs concitoyens, ne serait-ce qu'à titre conjoncturel. Pour reprendre un exemple cité, écrire que les électeurs boudent les urnes peut simplement pointer le dysfonctionnement de la démocratie représentative. Mais la teneur axiologique et moraliste du discours apparait plus crument quand le journaliste norme les attitudes et infantilise les abstentionnistes.

De même, la critique de l'incivisme est ostensible quand elle s'exprime par une adjectivation témoignant d'une crainte partagée cette fois avec d'autres acteurs que les citoyens : lorsque Jean-Marie Colombani dramatise l'enjeu en parlant de "désastre civique» (Le Monde, 27 septembre 1988), ou lorsque l'abstention est dite "alarmante» (La Tribune [Desfossés], 16 mars 1998), le discours journalistique se mêle à celui des politiques: Alain Juppé, par exemple, la juge «navrante, préoccupante» (Le Monde, 27 septembre 1988).

18. Voir Le Monde, 17-18 septembre 2000, et L'Humanité, 25 septembre 2000. 
Mais les propos politologiques courants sont-ils vraiment différents? Pour Dominique Reynié ("La dernière chance de l'ordre électoral », Le Figaro, 11 juin 2002), l'abstention « pose problème parce qu'elle exprime un mouvement de fond qui ne cesse pas d'être préoccupant». Même terminologie («plus préoccupant encore ») chez Anne Muxel, constatant que "seul un tiers des jeunes électeurs européens (...) ont voté [en 2004] » (2005, p. 50).

La dénonciation se fait plus sévère quand ce sont les citoyens « défaillants » qui sont seuls et directement visés. Pour ne retenir que quelques sentences, sans circonstances atténuantes, l'abstentionniste peut être lâche, irresponsable, léger, finalement coupable et sans vergogne : "La démocratie "suicidée" par des irresponsables! Voilà ce que m'évoque ce taux d'abstention » (courrier des lecteurs du Nouvel Observateur, 20 au 26 juin 2002); "La lâcheté commence par l'abstention » (André Diligent, dirigeant réformateur, cité par Le Progrès, 12 mars 1976) ; "L'insoutenable légèreté des abstentionnistes » (Laurent Joffrin, paraphrasant Kundera, "Les leçons d'un désastre », Le Nouvel Observateur, 25 avril 2002) ; " abstentionnistes, sans regrets et prêts à récidiver [...], sans remords ${ }^{19}$ sur leur attitude » (Gérard Courtois, Le Monde, 15 juin 2002).

En conclusion, ces associations récurrentes de l'abstention au pôle «négatif », qui résultent d'un long travail social d'imposition de la norme et d'inculcation du devoir électoral, ont donc produit dans le langage dominant une nette dichotomie et une remarquable superposition de clivages: abstention = anormal $=\mathrm{mal}=$ bas $=$ inactif et inerte $v s$ participation $=$ normal $=$ bien $=$ haut $=$ actif et mobile, etc.

Cette division manichéenne n'est pourtant qu'en partie intériorisée par les citoyens, si l'on en juge par l'évolution des taux d'abstention ou la persistance de discours allant à l'encontre de la doxa civique. Les anarchistes ont ainsi depuis longtemps renversé la métaphore dominante en faisant du vote la pathologie. Il est également remarquable que les porte-parole des partis recommandant exceptionnellement l'abstention éprouvent le besoin de la « positiver » en l'activant, perturbés de heurter les habitudes civiques de leurs électeurs. Ce fut le cas des communistes qui appelaient à l'abstention active au second tour de la présidentielle de 1969, ou des néo-gaullistes qui recommandaient la non participation active au référendum de 1998 sur la Nouvelle-Calédonie...

On aurait pu s'attendre, au moins, à ce que la science politique, (surtout dans ses livraisons scientifiques, sinon dans ses interventions politiques médiatisées), s'abstienne de jugements de valeur sur l'abstention. Nombre d'énoncés déjà cités - et d'autres plus moralistes encore - montrent le contraire. Serait-ce que les usages linguistiques sont inévitablement piégés et que tout discours,

19. Terme également utilisé par Anne Muxel (2003, p. 146) 
ici sur l'abstention, est d'emblée axiologisé ? En partie seulement, car rien n'oblige vraiment un énonciateur à parler de refuge, d'irresponsables, d'enfants gâtés ou de déserteurs, plutôt que de constater simplement une hausse de l'abstention. Et la norme est encore là, plus discrète, lorsqu'un dictionnaire politique contient des entrées élection, participation ou civisme mais pas abstention, ou lorsque des index des matières, et non des plus conventionnels, indiquent à l'entrée abstentionnisme électoral : «: (voir participation)».

Bien sûr, à repérer ces «creux», ces normes et ces anathèmes, on risque d'être soupçonné de promouvoir l'incivisme contre la doxa démocratique; d'être suspecté de faire l'apologie des régimes non pluralistes ou bien de défendre une conception "positiviste » de la science. Cependant notre propos ne vise pas, après tant de leçons de démocratie, à dénoncer ou à donner des leçons de méthode, mais simplement à souligner l'exigence du relativisme et de la réflexivité en matière scientifique (Offerlé, 1987 ; Lagroye, 1991, p. 17-18). À rappeler les potentialités moralisatrices et prescriptives du langage et à inviter à prendre conscience de nos propres présupposés normatifs.

Entre deux maux (être considéré comme "anti-démocrate" ou céder au conformisme ambiant qui, sur le mode de la lamentation convenue contre l'abstention, réunit les soirs d'élections politologues, journalistes et politiques), nous préférons renoncer aux "souhaits, exhortations, reproches et ordres» émaillant souvent le discours sur le monde social (Bourdieu, 1980, p.40), et prendre garde aux jugements de valeur, toujours susceptibles de se glisser... entre deux mots.

\section{Références}

\section{Dictionnaires}

LAROUSSE Pierre, 1866 [1990], Grand dictionnaire universel du XXe siècle, rééd. Lacour. Larousse universel en deux volumes, 1922, t. 1, Paris, Larousse.

Larousse du XXe siècle en sixvolumes, 1928, t. 1.

Grand Larousse encyclopédique (10 vol.), 1960, t. 1.

Grand dictionnaire encyclopédique Larousse, 1982, vol. 1.

REY-DEBOVE Josette, REY Alain, 1951, Le Robert, dictionnaire alphabétique et analogique

de la langue française, Paris, Presses universitaires de France.

— 1970, Le Robert, Le supplément, Paris, Presses universitaires de France.

— 1993, Le nouveau Petit Robert 1, Paris, Le Robert.

Trésor de la langue française (TLF), 1986, Paris, CNRS, Gallimard, vol. 1. 


\section{Ouvrages et articles}

BACOT Paul, 1994, Dictionnaire du vote. Élections et délibérations, Lyon, Presses universitaires de Lyon.

BOURDIEU Pierre, 1980, "Le sociologue en question », Questions de sociologie, Paris, Minuit.

BRAUD Philippe, 1980, Le suffrage universel contre la démocratie, Paris, Presses universitaires de France.

— 1996, L'émotion en politique, Paris, Presses de Sciences Po.

CAPDEVIELLE Jacques, 2005, Démocratie : la panne, Paris, Textuel, La Discorde.

CLANCHÉ François, 2003, "La participation électorale au printemps 2002. De plus en plus de votants intermittents ", Insee Première, $\mathrm{n}^{\circ} 877$, janvier.

DUCHESNE Sophie, 1994, «Engagement ou politique. Le paradoxe de la citoyenneté », P. Perrineau éd., L'engagement politique. Déclin ou mutation ?, Paris, Presses de la FNSP, p. 185-214.

GARRIGOU Alain, 2002, "L'abstention gagne les classes moyennes », Le Monde diplomatique, avril, p. 8-9.

GAXIE Daniel, 1978, Le cens caché. Inégalités culturelles et ségrégation politique, Paris, Le Seuil.

GOGUEL François, 1983, Chroniques électorales, vol. 2, Paris, Presses de la FNSP.

GRUNBERG Gérard, 1989, "La grève des urnes », Esprit, mars-avril, p. $124 \mathrm{~S}$.

GUENIFFEY Pascal, 1993, Le nombre et la raison. La révolution française et les élections, Paris, EHESS.

HONESTEMarie-Luce, 2004, «L'expression linguistique des représentations del'expérience (exemple de la localisation spatiale)», Actes du colloque "La polysémie», 17-19 novembre 2000, Presses de la Sorbonne.

LAGROYE Jacques, 1991, Sociologie politique, Paris, Presses de la FNSP et Dalloz.

LANCELOT Alain, 1968, Cahiers de la FNSP, n 162, L'abstentionnisme électoral en France, Paris, Armand Colin.

MEMMI Dominique, 1985, «L'engagement politique », M. Grawitz et J. Leca éd., Traité de science politique, Paris, Presses universitaires de France, t. 3.

MEYNAUD Jean, LANCELOT Alain, 1965, La participation des Français à la politique, Presses universitaires de France (Que sais-je ?), $2^{\mathrm{e}}$ édition.

MIRBEAU Octave, 1995 [1888], La grève des électeurs, Paris, Ludd.

MUXEL Anne, 2003, "La poussée des abstentions. Protestation, malaise, sanction», P. Perrineau et C. Ysmal éd. (voir infra).

— 2005, «Les abstentionnistes. Le premier parti européen », P. Perrineau et C. Ysmal éd., Le vote européen, de l'élargissement au référendum français, Paris, Presses de la FNSP, p. 45-76.

OFFERLÉ Michel, 2002 [1987], Les partis politiques, Paris, Presses universitaires de France (Que sais-je ?).

PERRINEAU Pascal et YSMAL Colette éd., 1995, Le vote de crise. L'élection présidentielle de 1995, Paris, Études politiques du Figaro / Presses de Sciences Po. 
— 2003, Le vote de tous les refus. Les élections présidentielle et législatives de 2002, Paris, Presses de Sciences Po.

PERRINEAU Pascal et REYNIÉ Dominique éd., 2001, Dictionnaire du vote, Paris, Presses universitaires de France.

RÉMI-GIRAUD Sylvianne, 2005, «France d'en haut / France d'en bas. Raffarin tout terrain », Mots. Les langages du politique, ${ }^{\circ}$ 77, p. 93-105.

SCHEMEIL Yves, 2001, « Le vote dans l'Ancien Orient », P. Perrineau et D. Reynié éd. (voir supra), p. 61-63.

SUBILEAU Françoise, 1992, Les facteurs de l'abstention, Paris, Cevipof, FNSP, multigraphié.

— 1996, «Le vote des Français à Maastricht. Le référendum comme procédure et le vote comme enjeu », Modern \& Contemporary France, Londres, New York, Addison Wesley Longman. 